\title{
Space-Dependent Dynamics in 1,4-Polybutadiene Nanocomposite
}

\author{
J. H. Roh, ${ }^{\dagger}$ M. Tyagi, ${ }^{\ddagger} \|$ T. E. Hogan, ${ }^{\S}$ and C. M. Roland ${ }^{* \dagger}$ \\ ${ }^{\dagger}$ Chemistry Division, Code 6120, Naval Research Laboratory, Washington, D.C. 20375-5342, United States \\ ${ }^{\ddagger}$ Center for Neutron Research, National Institute of Standards and Technology, Gaithersburg, Maryland 20899-6100, United States \\ ${ }^{\S}$ Center for Research and Technology, Bridgestone Americas, Akron, Ohio 44317-0001, United States \\ "Department of Materials Science and Engineering, University of Maryland, College Park, Maryland 20742, United States
}

$\mathrm{U}$ nderstanding the dynamics of the glass transition of polymers and molecular liquids has been a goal of physicists for more than a half-century. The nature of this transition is directly linked to the heterogeneity of the dynamics, reflected in spatial and temporal correlations of molecular motions, as well as a distribution of relaxation times. $^{1-4}$ The former can be quantified by a dynamic correlation length that grows on approaching the glassy state and the latter from the breadth of the relaxation function or dynamic susceptibility. The majority of experimental studies of this topic rely on dielectric spectroscopy, which provides relaxation spectra over many decades of frequency. However, the higher order susceptibilities involving both spatial and temporal correlations cannot be obtained from linear relaxation measurements without assumptions. ${ }^{5}$ Characterizing the spatial distribution of the dynamics requires other methods.

Since the 1990s quasi-elastic neutron scattering (QENS) has been widely employed to obtain space-specific information on the dynamic properties, providing momentum $(Q)$ and frequency dependences from the double differential scattering function of protons. ${ }^{6}$ The $Q$-dependent scattering from glassforming materials can be described using the KohlrauschWilliams-Watts (KWW) equation ${ }^{7-11}$

$$
\Phi_{\mathrm{KWW}}(Q, t) \propto \exp \left(-\left[t / \tau_{\alpha}(Q)\right]^{\beta(Q)}\right)
$$

For polymers $\tau_{\alpha}$ is the local segmental relaxation time, describing the time scale of correlated conformational transitions of backbone bonds. Note that the narrow dynamic range of neutron scattering introduces uncertainty in the value determined for the stretch exponent $\beta$. For small $Q$ in the range below the $Q$ value $\left(\sim 1 \AA^{-1}\right)$ for which the static structure factor shows its first maximum, $\tau_{\alpha}^{\beta} \sim Q^{-2},{ }^{12,13}$ the microscopic motions follow simple diffusion ("Gaussian approximation"). At larger $Q$ deviations from Gaussian behavior transpire, with the weaker wavevector dependence interpreted by a variety of models, including mode-coupling theory (MCT), jump diffusion models, and caging-decaging fractals. ${ }^{12,14,15}$ Molecular dynamics simulations have also proven useful in interpreting QENS experimental data. ${ }^{16}$

In this work we study the segmental dynamics of 1,4polybutadiene (PBD) in the presence of a high concentration of reinforcing filler particles. Reinforcement of rubbery polymers is especially intriguing because hard particles increase both the modulus and the failure strain (unlike plastics, which are made more brittle). Many applications of elastomers would not exist without the use of particulate reinforcement of the material. The PBD was synthesized by anionic polymerization (weight-average molecular weight $=158 \mathrm{kDa}$; polydispersity $=$
1.07 ; $14 \%$ vinyl repeat units; calorimetric $\left.T_{\mathrm{g}}=180 \mathrm{~K}\right)$. The carbon black was N110 (Cabot Vulcan 9), comprised of $17 \mathrm{~nm}$ particles fused into aggregates having a mean diameter of ca. 54 $\mathrm{nm}$. The aggregates have a highly structured surface, with an effective volume much larger than the actual volume. Carbon black has a heterogeneous topography, with local surface energies that can be as high as $100 \mathrm{~mJ} / \mathrm{m}^{2} \cdot{ }^{17} \mathrm{~N} 100$ is a furnace black with reactive double bonds at the particle surface that serve as binding sites for polymer chains. ${ }^{17}$ After mixing on a two-roll mill, the PBD composite samples were Soxhlet extracted with cyclohexane to remove unattached polymer. The initial composition of $20.5 \%$ by volume carbon increased to $60 \%$ on extraction (compositions determined by thermogravimetry). From the surface area of the carbon particles and their size and density, ${ }^{18}$ we calculate a specific surface area of $4.57 \times 10^{5} \mathrm{~cm}^{2}$ per $\mathrm{g}$ of composite (assuming no agglomeration during the extraction); this is about twice the specific volume of the PBD divided by its radius of gyration $\left(\sim 14.6 \mathrm{~nm}^{19,20}\right)$. Thus, for a single layer having a thickness equal to twice the radius of gyration, we estimate that only about a quarter of the particle surface is covered; moreover, every chain is adsorbed onto a particle since the material was extracted.

Interaction and binding of polymer segments to the particles constrain their motions increasing $\tau_{\alpha}{ }^{21}$ and molecular dynamics simulations have shown that interactions with nanoparticles increase the length scale of the cooperative motions of polymers underlying $\tau_{\alpha}{ }^{22}$ Interestingly, QENS of polyisoprene below its glass transition temperature showed increased librational mobility of the backbone in the presence of carbon black, ${ }^{23}$ although whether this result is specific to the glassy state remains to be investigated. More generally, the spatial variation of the constraints from particular fillers is unclear, and in order to explore this aspect of the dynamics, we employed quasielastic neutron scattering. Experiments were carried out using the high flux back-scattering spectrometer (HFBS, NG2) at the NIST Center for Neutron Research. The dynamic scattering function, $S(Q E)$, was measured with an energy resolution of $0.4 \mu \mathrm{eV}$ at up to $17 \mu \mathrm{eV}$; the $Q$ range was $0.31-1.71 \AA^{-1}$. A sample thickness of $0.1 \mathrm{~mm}$ was chosen to avoid multiple scattering. For our samples, we estimate the incoherent scattering cross section of hydrogen atoms in the polymers to be $94 \%$ of the total scattering for neat PBD and $82 \%$ for the extracted nanocomposite. Therefore, the scattering spectra reflect the energy-dependent distribution of selfcorrelation of the hydrogen atoms.

Received: August 1, 2013

Published: August 16, 2013 
The desired KWW function was expressed as a weighted superposition of Lorentzian functions

$$
\Phi_{\mathrm{KWW}}(\omega)=\int f(\log \tau) \frac{\omega \tau}{1+\omega^{2} \tau^{2}} \mathrm{~d}(\log \tau)
$$

For the distribution function, $f(\log \tau)$, we used the generalized version $^{24}$ of the expression of Rajagopal and Ngai. ${ }^{25}$ This model function was first convoluted with the experimental resolution function and then fit directly to the experimental $S(Q, \omega)$ to obtain $\tau_{\alpha}(Q)$ and $\beta(Q)$ at the various measurement temperatures.

Figure 1 shows master curves of the dynamic mechanical loss modulus, measured in shear using an Anton Paar MCR 502

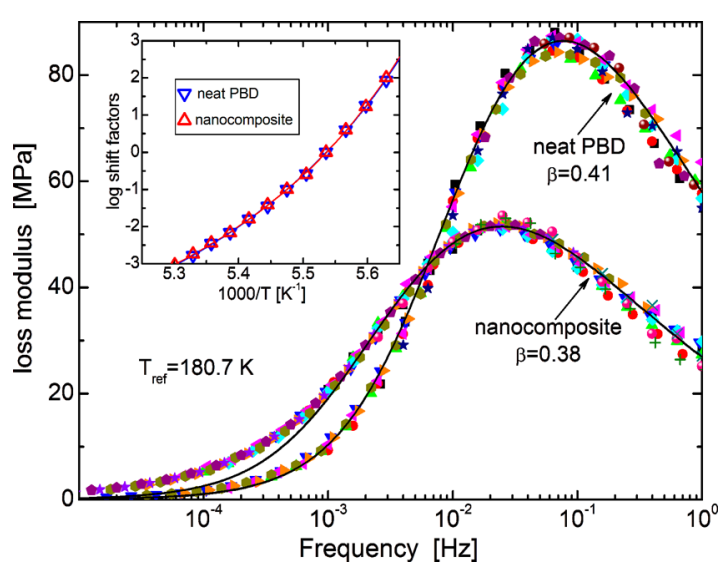

Figure 1. Master curves of the mechanical loss moduli of the PBD neat and with $20.5 \%$ by volume carbon particles along with the fitted KWW function. The inset shows the frequency-temperature shift factors, which have the same temperature dependence. Note for these measurements only, the filled sample was not extracted. Error bars are not larger than the symbol size.

rheometer, for PBD and the unextracted nanocomposite. The spectrum for the neat polymer is well-described by eq 1 with $\beta$ $=0.41$, which is in good agreement with the value determined by dielectric spectroscopy. ${ }^{26}$ Determining $\beta$ for the filled polymer is problematic. The mechanical loss peak in Figure 1 is significantly broader than for the neat PBD and can be approximately described using $\beta=0.38$, with deviation at lower frequencies. The presence of filler heterogeneously broadens the segmental relaxation because the effect of the carbon particles on the dynamics depends on the proximity of the segments to the particle. Of course, neither mechanical nor dielectric spectroscopy can provide information on this $Q$ dependence; the loss modulus measurements average over all length scales.

Figure 2 compares representative dynamic structure factors for the two materials at $T=300 \mathrm{~K} . S(E)$ for the nanocomposite is narrower, which indicates suppression of the dynamics. Fits of eq 2 to the spectra in Figure 2 are shown. For the neat PBD, $\beta=0.41$, with the $Q$-dependence of relaxation times shown in Figure 3a for four temperatures above $T_{\mathrm{g}}$. For $Q<1 \AA^{-1}$ the Gaussian approximation holds, $\tau_{\alpha}^{0.41} \sim Q^{-2}$. Note that the stretch exponent for the incoherent dynamic structure factor is $Q$-independent and thus is the same as $\beta$ measured by mechanical and dielectric spectroscopies. Included in Figure 3a are results from ref 13 for $Q<1 \AA^{-1}$; the agreement with the present data is satisfactory. At shorter length scales Fickian diffusion does not apply, and the Q-dependence becomes

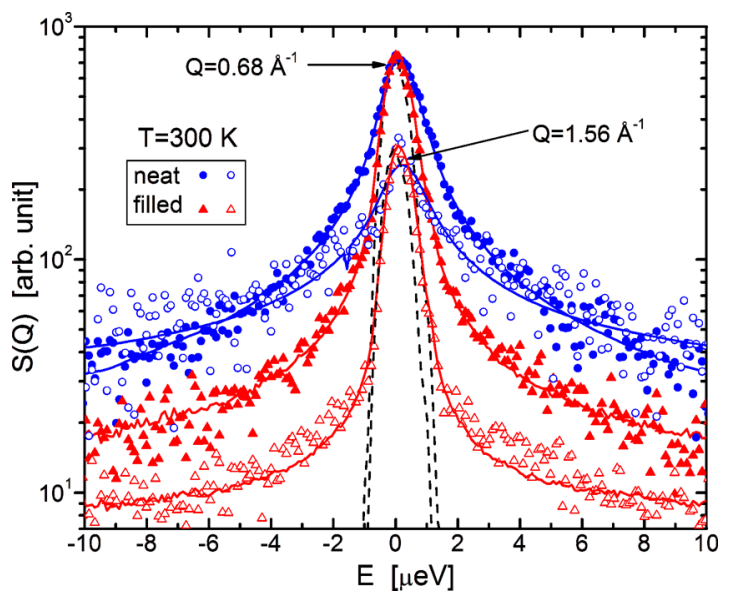

Figure 2. Incoherent dynamic structure factors at $300 \mathrm{~K}$ and the indicated wavevectors for neat PBD (circles) and the nanocomposite (triangles) along with fits to eq 2 (solid lines). The dashed lines indicate the experimental resolution measured at $4 \mathrm{~K}$.

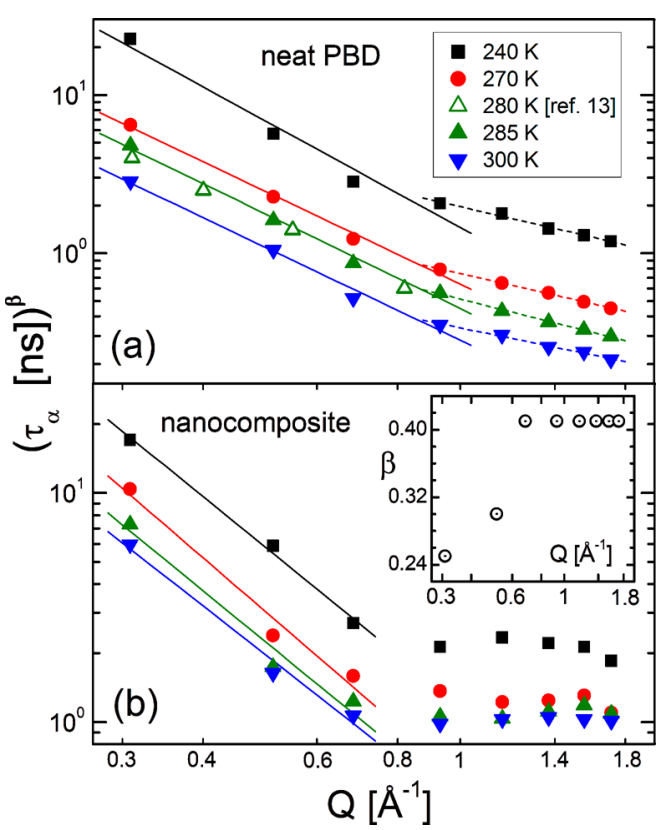

Figure 3. Local segmental relaxation times from QENS for (a) unfilled 1,4-polybutadiene, with the value of the stretch exponent obtained from mechanical spectroscopy (see Figure 1); (b) extracted nanocomposite with the $\beta(Q)$ (plotted in the inset) taken to be the values giving conformance to the Gaussian approximation for $<0.8 \AA^{-1}$ and fixed at 0.41 for higher $Q$. Lines are least-squares fits having a slope equal to -2 at small $Q$.

weaker, $\tau_{\alpha}^{0.41} \sim Q^{-0.9}$, indicating more local and/or isolated motions. The wavevector associated with this crossover in behavior is the same for all temperatures.

As noted, we cannot obtain from mechanical spectroscopy the value of $\beta(Q)$ for the nanocomposite; instead, we determine at each $Q$ the $\beta$ value yielding the expected $\tau_{\alpha} \sim$ $Q^{-2 / \beta}$ behavior for small $Q$ (recognizing that the $Q$ dependence of $\tau_{\alpha}$ may depend weakly on $\beta$ ). These results are shown in Figure $3 \mathrm{~b}$; note that the $\beta$ are independent of temperature. Similar to the results for the neat PBD, there is a transition to a very weak $Q$ dependence for larger $Q$ although this crossover occurs at a somewhat smaller wavevector (larger spatial range) than for neat $\mathrm{PBD}$. The $\beta(Q)$ for the nanocomposite are 
plotted versus $Q$ in the inset to Figure $3 b$. For larger $Q$ all segments are constrained by the particles, and the distribution of relaxation times is narrower than at larger length scales. The increase in the mean relaxation time due to the filler particles is larger at larger $Q$. For example, for the data in Figure 2, at $Q=$ $0.68 \AA^{-1} \tau_{\alpha}$ is about 6-fold larger for the nanocomposite compared to the neat PBD, whereas at $Q=1.56 \AA^{-1}$ the segmental relaxation increases a factor of 36 due to the filler particles.

In conclusion, binding of PBD chains to the carbon particle surface slows down the segmental motions and increases the heterogeneity of the dynamics, with the spectral stretching and change in $\tau_{\alpha}$ becoming spatially dependent; that is, the effect of the particles depends on the spatial distance of the segments, notwithstanding that all chains are attached to the particles.

\section{AUTHOR INFORMATION}

\section{Corresponding Author}

*E-mail mike.roland@nrl.navy.mil (C.M.R.).

\section{Notes}

The authors declare no competing financial interest.

\section{ACKNOWLEDGMENTS}

The work at NRL was supported by the Office of Naval Research. J.H.R. acknowledges a National Research Council postdoctoral fellowship. This work utilized facilities supported in part by the National Science Foundation under DMR0944772. The identification of commercial products does not imply endorsement by the National Institute of Standards and Technology nor does it imply that these are the best for the purpose.

\section{REFERENCES}

(1) Ediger, M. D. Annu. Rev. Phys. Chem. 2000, 51, 99-128.

(2) Sillescu, H.; Böhmer, R.; Diezemann, G.; Hinze, G. J. Non-Cryst. Solids 2002, 16, 307-310.

(3) Roland, C. M. Macromolecules 2010, 43, 7875-7890.

(4) Berthier, L., Biroli, G., Bouchaud, J.-P., Cipelletti, L., van Saarloos, W., Eds.; Dynamical Heterogeneities in Glasses, Colloids, and Granular Media; Oxford University Press: New York, 2011.

(5) Berthier, L.; Biroli, G.; Bouchaud, J.-P.; Kob, W.; Miyazaki, K.; Reichman, D. R. J. Chem. Phys. 2007, 126, 184503.

(6) Roland, C. M. Neutron Scattering in the Analysis of Polymers. In Encyclopedia of Analytical Chemistry; John Wiley \& Sons: New York, 2012.

(7) Zajac, W.; Gabrys, B. J.; McGreevy, R.; Mattssons, B. Physica B 1996, 226, 144-151.

(8) Richter, D.; Monkenbusch, M.; Arbe, A.; Colmenero, J. J. NonCryst. Solids 2001, 287, 286-296.

(9) Frick, B.; Dosseh, G.; Cailliaux, A.; Alba-Simionesco, C. Chem. Phys. 2003, 292, 311-323.

(10) Busselez, R.; Lefort, R.; Ghoufi, A.; Beuneu, B.; Frick, B.; Affouard, F.; Morineau, D. J. Phys.: Condens. Matter 2011, 23, 505102.

(11) Arrighi, V.; Tanchawanich, J.; Telling, M.T. F. Macromolecules 2013, 46, 216-225.

(12) Arbe, A.; Colmenero, J.; Alvarez, F.; Mokenbusch, M.; Richter, D.; Farago, B.; Frick, B. Phys. Rev. E 2003, 67, 051802.

(13) Arbe, A.; Colmenero, J.; Monkenbusch, M.; Richter, D. Phys. Rev. Lett. 1998, 81, 590-593.

(14) Zorn, R. Phys. Rev. B 1997, 55, 6249-6259.

(15) Vorselaars, B.; Lyulin, A. V.; Karatasos, K.; Michels, M. A. J. Phys. Rev. E 2007, 75, 011504.

(16) Colmenero, J.; Brodeck, M.; Arbe, A.; Richter, D. Macromolecules 2013, 46, 1478-1485.
(17) Donnet, J.-P.; Custodero, E. In The Science and Technology of Rubber, 4th ed.; Mark, J. E., Erman, B., Roland, C. M., Eds.; Academic Press: New York, 2013.

(18) Hess, W. M.; McDonald, G. C. Rubber Chem. Technol. 1983, 56, 892-917.

(19) Fetters, L. J.; Hadjichristidis, N.; Linder, J. S.; Mays, J. W. J. Phys. Chem. Ref. Data 1994, 23, 619-640.

(20) Flory, P. J. Statistical Mechanics of Chain Molecules; Oxford University Press: New York, 1989.

(21) Robertson, C. G.; Roland, C. M. Rubber Chem. Technol. 2008, $81,506-522$.

(22) Starr, F. W.; Douglas, J. F. Phys. Rev. Lett. 2011, 106, 115702.

(23) Nakatani, A. I.; Ivkov, R.; Papanek, P.; Yang, H.; Gersphacher, M. Rubber Chem. Technol. 2000, 73, 847-863.

(24) Gomez, D.; Alegria, A. J. Non-Cryst. Solids 2001, 287, 246-251.

(25) Rajagopal, A. K.; Ngai, K. L. In Relaxations in Complex Systems; Ngai, K. L., Wright, G. B., Eds.; North Holland: Amsterdam, 1991.

(26) Arbe, A.; Richter, D.; Colmenero, J.; Farago, B. Phys. Rev. E 1996, 54, 3853-3869. 